\title{
A note on reverses of Young type inequalities
}

\author{
Xingkai $\mathrm{Hu}^{1 *}$ and Jianming $\mathrm{Xue}^{2}$
}

"Correspondence:

huxingkai84@163.com

'Faculty of Science, Kunming

University of Science and

Technology, Kunming, Yunnan 650500, P.R. China

Full list of author information is

available at the end of the article

\section{Abstract}

In this paper, we obtain some improved reverses of Young type inequalities which were established by Burqan and Khandaqji (J. Math. Inequal. 9:113-120, 2015).

MSC: $15 \mathrm{~A} 45 ; 15 \mathrm{~A} 60$

Keywords: unitarily invariant norms; Young type inequalities; positive semidefinite matrices; singular values

\section{Introduction}

Let $M_{n}$ be the space of $n \times n$ complex matrices. Let $\|\cdot\|$ denote any unitarily invariant norm on $M_{n}$. So, $\|U A V\|=\|A\|$ for all $A \in M_{n}$ and for all unitary matrices $U, V \in M_{n}$. For $A=\left[a_{i j}\right] \in M_{n}$, the Hilbert-Schmidt norm and the trace norm of $A$ are defined by $\|A\|_{2}=\sqrt{\sum_{j=1}^{n} s_{j}^{2}(A)},\|A\|_{1}=\sum_{j=1}^{n} s_{j}(A)$, respectively, where $s_{i}(A)(i=1, \ldots, n)$ are the singular values of $A$ with $s_{1}(A) \geq \cdots \geq s_{n}(A)$, which are the eigenvalues of the positive semidefinite matrix $|A|=\left(A A^{*}\right)^{\frac{1}{2}}$, arranged in decreasing order and repeated according to multiplicity.

The classical Young inequality says that if $a, b \geq 0$ and $0 \leq v \leq 1$, then

$$
a^{v} b^{1-v} \leq v a+(1-v) b
$$

with equality if and only if $a=b$.

Kittaneh and Manasrah [1] obtained an improvement of inequality (1) which can be stated as follows:

$$
a^{v} b^{1-v}+r_{0}(\sqrt{a}-\sqrt{b})^{2} \leq v a+(1-v) b,
$$

where $r_{0}=\min \{v, 1-v\}$.

Recently, Burqan and Khandaqji [2] gave the following reverses of the scalar Young type inequalities:

$$
v^{2} a^{2}+(1-v)^{2} b^{2} \leq(1-v)^{2}(a-b)^{2}+a^{2 v}[(1-v) b]^{2-2 v}, \quad 0 \leq v \leq \frac{1}{2},
$$

and

$$
v^{2} a^{2}+(1-v)^{2} b^{2} \leq v^{2}(a-b)^{2}+(v a)^{2 v} b^{2-2 v}, \quad \frac{1}{2} \leq v \leq 1 .
$$

(C) 2015 Hu and Xue; licensee Springer. This is an Open Access article distributed under the terms of the Creative Commons Attribution License (http://creativecommons.org/licenses/by/4.0), which permits unrestricted use, distribution, and reproduction in any medium, provided the original work is properly credited. 
A matrix Young inequality, proved in [3], says that if $A, B \in M_{n}$ are positive semidefinite, then

$$
s_{j}\left(A^{v} B^{1-v}\right) \leq s_{j}(v A+(1-v) B)
$$

for $j=1, \ldots, n$.

Based on the reverses of the scalar Young type inequalities (3) and (4), Burqan and Khandaqji proved the following in [2] if $A, B, X \in M_{n}$ such that $A$ and $B$ are positive semidefinite. If $0 \leq v \leq \frac{1}{2}$, then

$$
\begin{aligned}
& \|v A X+(1-v) X B\|_{2}^{2} \\
& \quad \leq(1-v)^{2}\|A X-X B\|_{2}^{2}+2 v(1-v)\left\|A^{\frac{1}{2}} X B^{\frac{1}{2}}\right\|_{2}^{2}+(1-v)^{2(1-v)}\left\|A^{v} X B^{1-v}\right\|_{2}^{2} .
\end{aligned}
$$

If $\frac{1}{2} \leq v \leq 1$, then

$$
\|v A X+(1-v) X B\|_{2}^{2} \leq v^{2}\|A X-X B\|_{2}^{2}+2 v(1-v)\left\|A^{\frac{1}{2}} X B^{\frac{1}{2}}\right\|_{2}^{2}+v^{2 v}\left\|A^{v} X B^{1-v}\right\|_{2}^{2} .
$$

At the same time, Burqan and Khandaqji proved the following in [2] if $A, B \in M_{n}$ such that $A$ and $B$ are positive semidefinite. If $0 \leq v \leq \frac{1}{2}$, then

$$
\begin{aligned}
& (1-v)^{1-v}\left\|A^{v}\right\|_{2}\left\|B^{1-v}\right\|_{2} \\
& \quad \geq \sqrt{v^{2}\|A\|_{2}^{2}+(1-v)^{2}\|B\|_{2}^{2}-(1-v)^{2}\left(\|A\|_{2}^{2}+\|B\|_{2}^{2}-2\|A B\|_{1}\right)} .
\end{aligned}
$$

If $\frac{1}{2} \leq v \leq 1$, then

$$
v^{v}\left\|A^{v}\right\|_{2}\left\|B^{1-v}\right\|_{2} \geq \sqrt{v^{2}\|A\|_{2}^{2}+(1-v)^{2}\|B\|_{2}^{2}-v^{2}\left(\|A\|_{2}^{2}+\|B\|_{2}^{2}-2\|A B\|_{1}\right)} .
$$

For more information on matrix versions of the Young inequality (1) the reader is referred to [4-9].

The main purpose of this paper is to give improved reverses of Young type inequalities (3) and (4). Then we use these inequalities to establish corresponding inequalities for matrices. To achieve our goal we need the following reverses of Young type inequalities for scalars.

\section{Reverses of Young type inequalities for scalars}

We begin this section with the reverses of Young type inequalities for scalars.

Theorem 1 Let $a, b \geq 0$. If $0 \leq v \leq \frac{1}{2}$, then

$$
v^{2} a^{2}+(1-v)^{2} b^{2}+r_{0} a(\sqrt{(1-v) b}-\sqrt{a})^{2} \leq(1-v)^{2}(a-b)^{2}+a^{2 v}[(1-v) b]^{2-2 v},
$$

where $r_{0}=\min \{2 v, 1-2 v\}$.

$$
\text { If } \frac{1}{2} \leq v \leq 1 \text {, then }
$$

$$
v^{2} a^{2}+(1-v)^{2} b^{2}+r_{0} b(\sqrt{b}-\sqrt{v a})^{2} \leq v^{2}(a-b)^{2}+(v a)^{2 v} b^{2-2 v},
$$

where $r_{0}=\min \{2 v-1,2-2 v\}$. 
Proof If $0 \leq v \leq \frac{1}{2}$, then, by inequality (2), we have

$$
\begin{aligned}
(1-v)^{2}(a-b)^{2}-v^{2} a^{2}-(1-v)^{2} b^{2}-r_{0} a(\sqrt{(1-v) b}-\sqrt{a})^{2}+a^{2 v}[(1-v) b]^{2-2 v} \\
=a[(1-2 v) a+2 v(1-v) b]-r_{0} a(\sqrt{(1-v) b}-\sqrt{a})^{2}-2(1-v) a b+a^{2 v}[(1-v) b]^{2-2 v} \\
\geq a\left\{a^{1-2 v}[(1-v) b]^{2 v}\right\}-2(1-v) a b+a^{2 v}[(1-v) b]^{2-2 v} \\
=a^{2-2 v}[(1-v) b]^{2 v}+a^{2 v}[(1-v) b]^{2-2 v}-2(1-v) a b \\
=\left[a^{1-v}(1-v)^{v} b^{v}-a^{v}(1-v)^{1-v} b^{1-v}\right]^{2} \geq 0,
\end{aligned}
$$

and so

$$
v^{2} a^{2}+(1-v)^{2} b^{2}+r_{0} a(\sqrt{(1-v) b}-\sqrt{a})^{2} \leq(1-v)^{2}(a-b)^{2}+a^{2 v}[(1-v) b]^{2-2 v} .
$$

If $\frac{1}{2} \leq v \leq 1$, then

$$
\begin{aligned}
v^{2} & (a-b)^{2}-v^{2} a^{2}-(1-v)^{2} b^{2}-r_{0} b(\sqrt{b}-\sqrt{v a})^{2}+(v a)^{2 v} b^{2-2 v} \\
& =(2 v-1) b^{2}+(2-2 v) v a b-r_{0} b(\sqrt{b}-\sqrt{v a})^{2}-2 v a b+(v a)^{2 v} b^{2-2 v} \\
& =b\left[(2 v-1) b+(2-2 v) v a-r_{0}(\sqrt{b}-\sqrt{v a})^{2}\right]-2 v a b+(v a)^{2 v} b^{2-2 v} \\
& \geq b\left[b^{2 v-1}(v a)^{2-2 v}\right]-2 v a b+(v a)^{2 v} b^{2-2 v} \\
& =\left[b^{v}(v a)^{1-v}-(v a)^{v} b^{1-v}\right]^{2} \geq 0,
\end{aligned}
$$

and so

$$
v^{2} a^{2}+(1-v)^{2} b^{2}+r_{0} b(\sqrt{b}-\sqrt{v a})^{2} \leq v^{2}(a-b)^{2}+(v a)^{2 v} b^{2-2 v} .
$$

This completes the proof.

Remark 1 Obviously, (9) and (10) are improvement reverses of the scalar Young type inequalities (3) and (4).

\section{Reverses of Young type inequalities for matrices}

Based on the reverses of the scalar Young type inequalities (9) and (10), we obtain matrix versions of these inequalities.

Theorem 2 Let $A, B, X \in M_{n}$ such that $A$ and $B$ are positive semidefinite. If $0 \leq v \leq \frac{1}{2}$, then

$$
\begin{gathered}
\|v A X+(1-v) X B\|_{2}^{2}+r_{0}\left[(1-v)\left\|A^{\frac{1}{2}} X B^{\frac{1}{2}}\right\|_{2}^{2}+\|A X\|_{2}^{2}-2 \sqrt{1-v}\left\|A^{\frac{3}{4}} X B^{\frac{1}{4}}\right\|_{2}^{2}\right] \\
\leq(1-v)^{2}\|A X-X B\|_{2}^{2}+2 v(1-v)\left\|A^{\frac{1}{2}} X B^{\frac{1}{2}}\right\|_{2}^{2}+(1-v)^{2(1-v)}\left\|A^{v} X B^{1-v}\right\|_{2}^{2},
\end{gathered}
$$

where $r_{0}=\min \{2 v, 1-2 v\}$.

$$
\text { If } \frac{1}{2} \leq v \leq 1 \text {, then }
$$

$$
\begin{gathered}
\|v A X+(1-v) X B\|_{2}^{2}+r_{0}\left[v\left\|A^{\frac{1}{2}} X B^{\frac{1}{2}}\right\|_{2}^{2}+\|X B\|_{2}^{2}-2 \sqrt{v}\left\|A^{\frac{1}{4}} X B^{\frac{3}{4}}\right\|_{2}^{2}\right] \\
\leq v^{2}\|A X-X B\|_{2}^{2}+2 v(1-v)\left\|A^{\frac{1}{2}} X B^{\frac{1}{2}}\right\|_{2}^{2}+v^{2 v}\left\|A^{v} X B^{1-v}\right\|_{2}^{2}
\end{gathered}
$$

where $r_{0}=\min \{2 v-1,2-2 v\}$. 
Proof Since every positive semidefinite matrix is unitarily diagonalizable, it follows that there are unitary matrices $U, V \in M_{n}$ such that $A=U D U^{*}$ and $B=V E V^{*}$, where

$$
D=\operatorname{diag}\left(\lambda_{1}, \ldots, \lambda_{n}\right), \quad E=\operatorname{diag}\left(\mu_{1}, \ldots, \mu_{n}\right), \quad \text { and } \lambda_{i}, \mu_{i} \geq 0, i=1, \ldots, n \text {. }
$$

Let $Y=U^{*} X V=\left[y_{i j}\right]$. Then

$$
\begin{aligned}
& v A X+(1-v) X B=U(v D Y+(1-v) Y E) V^{*}=U\left[\left(v \lambda_{i}+(1-v) \mu_{j}\right) y_{i j}\right] V^{*}, \\
& A X-X B=U\left[\left(\lambda_{i}-\mu_{j}\right) y_{i j}\right] V^{*}, \quad A^{\frac{1}{2}} X B^{\frac{1}{2}}=U\left[\lambda_{i}^{\frac{1}{2}} \mu_{j}^{\frac{1}{2}} y_{i j}\right] V^{*},
\end{aligned}
$$

and

$$
A^{v} X B^{1-v}=U\left[\lambda_{i}^{v} \mu_{j}^{1-v} y_{i j}\right] V^{*}
$$

If $0 \leq v \leq \frac{1}{2}$, by inequality (9), we have

$$
\begin{aligned}
\| v A X & +(1-v) X B \|_{2}^{2} \\
= & \sum_{i, j=1}^{n}\left(v \lambda_{i}+(1-v) \mu_{j}\right)^{2}\left|y_{i j}\right|^{2} \\
\leq & (1-v)^{2} \sum_{i, j=1}^{n}\left(\lambda_{i}-\mu_{j}\right)^{2}\left|y_{i j}\right|^{2}+(1-v)^{2(1-v)} \sum_{i, j=1}^{n}\left(\lambda_{i}^{v} \mu_{j}^{1-v}\right)^{2}\left|y_{i j}\right|^{2} \\
& -r_{0} \sum_{i, j=1}^{n} \lambda_{i}\left(\sqrt{(1-v) \mu_{j}}-\sqrt{\lambda_{i}}\right)^{2}\left|y_{i j}\right|^{2}+2 v(1-v) \sum_{i, j=1}^{n}\left(\lambda_{i}^{\frac{1}{2}} \mu_{j}^{\frac{1}{2}}\right)^{2}\left|y_{i j}\right|^{2} \\
= & (1-v)^{2} \sum_{i, j=1}^{n}\left(\lambda_{i}-\mu_{j}\right)^{2}\left|y_{i j}\right|^{2}+(1-v)^{2(1-v)} \sum_{i, j=1}^{n}\left(\lambda_{i}^{v} \mu_{j}^{1-v}\right)^{2}\left|y_{i j}\right|^{2} \\
& +\left(2 v-r_{0}\right)(1-v) \sum_{i, j=1}^{n}\left(\lambda_{i}^{\frac{1}{2}} \mu_{j}^{\frac{1}{2}}\right)^{2}\left|y_{i j}\right|^{2} \\
& -r_{0} \sum_{i, j=1}^{n} \lambda_{i}^{2}\left|y_{i j}\right|^{2}+2 r_{0} \sqrt{(1-v)} \sum_{i, j=1}^{n}\left(\lambda_{i}^{\frac{3}{4}} \mu_{j}^{\frac{1}{4}}\right)^{2}\left|y_{i j}\right|^{2} \\
= & (1-v)^{2}\|A X-X B\|_{2}^{2}+(1-v)^{2(1-v)}\left\|A^{v} X B^{1-v}\right\|_{2}^{2} \\
& +\left(2 v-r_{0}\right)(1-v)\left\|A^{\frac{1}{2}} X B^{\frac{1}{2}}\right\|_{2}^{2}-r_{0}\|A X\|_{2}^{2}+2 r_{0} \sqrt{1-v}\left\|A^{\frac{3}{4}} X B^{\frac{1}{4}}\right\|_{2}^{2},
\end{aligned}
$$

and so

$$
\begin{gathered}
\|v A X+(1-v) X B\|_{2}^{2}+r_{0}\left[(1-v)\left\|A^{\frac{1}{2}} X B^{\frac{1}{2}}\right\|_{2}^{2}+\|A X\|_{2}^{2}-2 \sqrt{1-v}\left\|A^{\frac{3}{4}} X B^{\frac{1}{4}}\right\|_{2}^{2}\right] \\
\leq(1-v)^{2}\|A X-X B\|_{2}^{2}+2 v(1-v)\left\|A^{\frac{1}{2}} X B^{\frac{1}{2}}\right\|_{2}^{2}+(1-v)^{2(1-v)}\left\|A^{v} X B^{1-v}\right\|_{2}^{2} .
\end{gathered}
$$

If $\frac{1}{2} \leq v \leq 1$, then by inequality (10) and the same method above, we have inequality (12). This completes the proof. 
Remark 2 Obviously, (11) and (12) are improvement reverses of the matrix Young type inequalities (5) and (6).

In the end, we present two new inequalities, by means of inequalities (9) and (10). To do this, we need the following lemmas.

Lemma 1 (Cauchy-Schwarz inequality) [10] Let $a_{i} \geq 0, b_{i} \geq 0$, for $i=1,2, \ldots, n$, then

$$
\sum_{i=1}^{n} a_{i} b_{i} \leq\left(\sum_{i=1}^{n} a_{i}^{2}\right)^{\frac{1}{2}}\left(\sum_{i=1}^{n} b_{i}^{2}\right)^{\frac{1}{2}} .
$$

Lemma 2 [10] Let $A, B \in M_{n}$, then

$$
\sum_{j=1}^{n} s_{j}(A B) \leq \sum_{j=1}^{n} s_{j}(A) s_{j}(B) .
$$

Theorem 3 Let $A, B \in M_{n}$ such that $A$ and $B$ are positive semidefinite. If $0 \leq v \leq \frac{1}{2}$, then

$$
\begin{aligned}
& (1-v)^{1-v}\left\|A^{v}\right\|_{2}\left\|B^{1-v}\right\|_{2} \\
& \geq \sqrt{v^{2}\|A\|_{2}^{2}+(1-v)^{2}\|B\|_{2}^{2}-(1-v)^{2}\left(\|A\|_{2}^{2}+\|B\|_{2}^{2}-2\|A B\|_{1}\right)+M_{1}},
\end{aligned}
$$

where $r_{0}=\min \{2 v, 1-2 v\}, M_{1}=r_{0}\left[(1-v)\|A B\|_{1}+\|A\|_{2}^{2}-2 \sqrt{1-v}\left\|A^{\frac{3}{2}}\right\|_{1}\left\|B^{\frac{1}{2}}\right\|_{1}\right]$.

$$
\text { If } \frac{1}{2} \leq v \leq 1 \text {, then }
$$

$$
v^{v}\left\|A^{v}\right\|_{2}\left\|B^{1-v}\right\|_{2} \geq \sqrt{v^{2}\|A\|_{2}^{2}+(1-v)^{2}\|B\|_{2}^{2}-v^{2}\left(\|A\|_{2}^{2}+\|B\|_{2}^{2}-2\|A B\|_{1}\right)+M_{2}},
$$

where $r_{0}=\min \{2 v-1,2-2 v\}, M_{2}=r_{0}\left[v\|A B\|_{1}+\|B\|_{2}^{2}-2 \sqrt{v}\left\|A^{\frac{1}{2}}\right\|_{1}\left\|B^{\frac{3}{2}}\right\|_{1}\right]$.

Proof If $0 \leq v \leq \frac{1}{2}$, then using Lemma 1, Lemma 2, and inequality (9), we have

$$
\begin{aligned}
\operatorname{tr} & \left(v^{2} A^{2}+(1-v)^{2} B^{2}\right) \\
= & v^{2} \operatorname{tr} A^{2}+(1-v)^{2} \operatorname{tr} B^{2} \\
= & \sum_{j=1}^{n}\left(v^{2} s_{j}^{2}(A)+(1-v)^{2} s_{j}^{2}(B)\right) \\
\leq & (1-v)^{2}\left[\sum_{j=1}^{n} s_{j}^{2}(A)+\sum_{j=1}^{n} s_{j}^{2}(B)-2 \sum_{j=1}^{n} s_{j}(A) s_{j}(B)\right] \\
& +\sum_{j=1}^{n}(1-v)^{2(1-v)}\left[s_{j}\left(A^{v}\right) s_{j}\left(B^{1-v}\right)\right]^{2}-r_{0} \sum_{j=1}^{n} s_{j}(A)\left[\sqrt{(1-v) s_{j}(B)}-\sqrt{s_{j}(A)}\right]^{2} \\
\leq & (1-v)^{2}\left[\|A\|_{2}^{2}+\|B\|_{2}^{2}-2 \sum_{j=1}^{n} s_{j}(A B)\right]+(1-v)^{2(1-v)}\left[\sum_{j=1}^{n} s_{j}\left(A^{v}\right) s_{j}\left(B^{1-v}\right)\right]^{2} \\
& \quad-r_{0}\left[(1-v) \sum_{j=1}^{n} s_{j}(A) s_{j}(B)+\sum_{j=1}^{n} s_{j}^{2}(A)-2 \sqrt{1-v}\left(\sum_{j=1}^{n} s_{j}^{\frac{3}{2}}(A) s_{j}^{\frac{1}{2}}(B)\right)\right]
\end{aligned}
$$




$$
\begin{aligned}
\leq & (1-v)^{2}\left[\|A\|_{2}^{2}+\|B\|_{2}^{2}-2\|A B\|_{1}\right]+(1-v)^{2(1-v)}\left[\sum_{j=1}^{n} s_{j}^{2}\left(A^{v}\right) \sum_{j=1}^{n} s_{j}^{2}\left(B^{1-v}\right)\right] \\
& -r_{0}\left[(1-v) \sum_{j=1}^{n} s_{j}(A B)+\|A\|_{2}^{2}-2 \sqrt{1-v}\left(\sum_{j=1}^{n} s_{j}^{\frac{3}{4}}(A) s_{j}^{\frac{1}{4}}(B)\right)^{2}\right] \\
\leq & (1-v)^{2}\left[\|A\|_{2}^{2}+\|B\|_{2}^{2}-2\|A B\|_{1}\right]+(1-v)^{2(1-v)}\left\|A^{v}\right\|_{2}^{2}\left\|B^{1-v}\right\|_{2}^{2} \\
& -r_{0}\left[(1-v)\|A B\|_{1}+\|A\|_{2}^{2}-2 \sqrt{1-v}\left(\sum_{j=1}^{n} s_{j}^{\frac{3}{2}}(A) \sum_{j=1}^{n} s_{j}^{\frac{1}{2}}(B)\right)\right] .
\end{aligned}
$$

Thus

$$
\begin{aligned}
v^{2}\|A\|_{2}^{2}+(1-v)^{2}\|B\|_{2}^{2} \leq & (1-v)^{2}\left(\|A\|_{2}^{2}+\|B\|_{2}^{2}-2\|A B\|_{1}\right)+(1-v)^{2(1-v)}\left\|A^{v}\right\|_{2}^{2}\left\|B^{1-v}\right\|_{2}^{2} \\
& -r_{0}\left[(1-v)\|A B\|_{1}+\|A\|_{2}^{2}-2 \sqrt{1-v}\left\|A^{\frac{3}{2}}\right\|_{1}\left\|B^{\frac{1}{2}}\right\|_{1}\right] .
\end{aligned}
$$

If $\frac{1}{2} \leq v \leq 1$, then by inequality (10) and the same method above, we have inequality (14). This completes the proof.

Remark 3 It should be noticed that neither (7) nor (13) is uniformly better than the other. At the same time, neither (8) nor (14) is uniformly better than the other.

\section{Competing interests}

The authors declare that they have no competing interests.

\section{Authors' contributions}

All authors contributed equally to the writing of this paper. All authors read and approved the final manuscript.

\section{Author details}

${ }^{1}$ Faculty of Science, Kunming University of Science and Technology, Kunming, Yunnan 650500, P.R. China. ${ }^{2}$ Oxbridge College, Kunming University of Science and Technology, Kunming, Yunnan 650106, P.R. China.

\section{Acknowledgements}

The authors wish to express their heartfelt thanks to the referees for their detailed and helpful suggestions for revising the manuscript.

Received: 30 November 2014 Accepted: 6 March 2015 Published online: 17 March 2015

\section{References}

1. Kittaneh, F, Manasrah, Y: Improved Young and Heinz inequalities for matrices. J. Math. Anal. Appl. 361, 262-269 (2010)

2. Burqan, A, Khandaqji, M: Reverses of Young type inequalities. J. Math. Inequal. 9, 113-120 (2015)

3. Ando, T: Matrix Young inequalities. Oper. Theory, Adv. Appl. 75, 33-38 (1995)

4. Bhatia, R, Kittaneh, F: On singular values of a product of operators. SIAM J. Matrix Anal. Appl. 11, 271-277 (1990)

5. Hu, X: Young type inequalities for matrices. J. East China Norm. Univ. Natur. Sci. Ed. 4, 12-17 (2012)

6. Zhan, X: Inequalities for unitarily invariant norms. SIAM J. Matrix Anal. Appl. 20, $466-470$ (1998)

7. Peng, Y: Young type inequalities for matrices. Ital. J. Pure Appl. Math. 32, 515-518 (2014)

8. Kittaneh, F, Manasrah, Y: Reverse Young and Heinz inequalities for matrices. Linear Multilinear Algebra 59, 1031-1037 (2011)

9. He, C, Zou, L: Some inequalities involving unitarily invariant norms. Math. Inequal. Appl. 15, 767-776 (2012)

10. Bhatia, R: Matrix Analysis. Springer, New York (1997) 